\title{
Introduction to Electroweak Symmetry Breaking
}

\author{
S. Dawson
}

Department of Physics, Brookhaven National Laboratory, Upton, NY 11973-5000

\author{
Presented at the \\ XIII Mexican School of Particles and Fields \\ Sonora, Mexico \\ October 2-11, 2008
}

December 2008

\section{Physics Department \\ Brookhaven National Laboratory \\ P.O. Box 5000 \\ Upton, NY 11973-5000 \\ www.bnl.gov}

\begin{abstract}
Notice: This manuscript has been authored by employees of Brookhaven Science Associates, LLC under Contract No. DE-AC02-98CH10886 with the U.S. Department of Energy. The publisher by accepting the manuscript for publication acknowledges that the United States Government retains a non-exclusive, paid-up, irrevocable, world-wide license to publish or reproduce the published form of this manuscript, or allow others to do so, for United States Government purposes.
\end{abstract}

This preprint is intended for publication in a journal or proceedings. Since changes may be made before publication, it may not be cited or reproduced without the author's permission. 


\section{DISCLAIMER}

This report was prepared as an account of work sponsored by an agency of the United States Government. Neither the United States Government nor any agency thereof, nor any of their employees, nor any of their contractors, subcontractors, or their employees, makes any warranty, express or implied, or assumes any legal liability or responsibility for the accuracy, completeness, or any third party's use or the results of such use of any information, apparatus, product, or process disclosed, or represents that its use would not infringe privately owned rights. Reference herein to any specific commercial product, process, or service by trade name, trademark, manufacturer, or otherwise, does not necessarily constitute or imply its endorsement, recommendation, or favoring by the United States Government or any agency thereof or its contractors or subcontractors. The views and opinions of authors expressed herein do not necessarily state or reflect those of the United States Government or any agency thereof. 


\title{
Introduction to Electroweak Symmetry Breaking'
}

\author{
S. Dawson \\ Physics Department, Brookhaven National Laboratory \\ Upton, New York 11973
}

\begin{abstract}
.
In these lectures, I review the status of the electroweak sector of the Standard Model, with an emphasis on the importance of radiative corrections and searches for the Standard Model Higgs boson. A discussion of the special role of the $\mathrm{TeV}$ energy scale in electroweak physics is included.
\end{abstract}

Keywords: Higgs boson, Standard Model

PACS: $12.15 .-\mathrm{y}, 14.80 . \mathrm{Bn}$

\section{INTRODUCTION}

The Standard Model (SM) is the backbone of elementary particle physics-not only does it provide a consistent framework for studying the interactions of quark and leptons, but it also gives predictions which have been extensively tested experimentally. In these notes, I review the electroweak sector of the Standard Model, discuss the calculation of electroweak radiative corrections to observables, and summarize the status of SM Higgs boson searches.

Despite the impressive experimental successes, however, the electroweak theory is not completely satisfactory and the mechanism of electroweak symmetry breaking is untested. I will discuss the logic behind the oft-repeated statement: "There must be new physics at the $\mathrm{TeV}$ scale". These lectures reflect my strongly held belief that upcoming results from the LHC will fundamentally change our understanding of electroweak symmetry breaking.

\section{THE STANDARD MODEL}

The electroweak sector of the Standard Model has been reviewed extensively in the literature $[1,2,3,4,5,6,7,8]$ and I provide only a brief summary here. The WeinbergSalam model is an $S U(2)_{L} \times U(1)_{Y}$ gauge theory containing three $S U(2)_{L}$ gauge bosons, $W_{\mu}^{i}, i=1,2,3$, and one $U(1)_{Y}$ gauge boson, $B_{\mu}$, with kinetic energy terms,

$$
\mathscr{L}_{\mathrm{KE}}=-\frac{1}{4} \Sigma_{i=1}^{3} W_{\mu \nu}^{i} W^{\mu v i}-\frac{1}{4} B_{\mu v} B^{\mu \nu}
$$

\footnotetext{
${ }^{1}$ Lectures given at the XIII Mexican School of Particles and Fields, 2-11 October, 2008, Sonora, Mexico.
} 
where

$$
\begin{aligned}
W_{\mu v}^{i} & =\partial_{\nu} W_{\mu}^{i}-\partial_{\mu} W_{v}^{i}-g \varepsilon^{i j k} W_{\mu}^{j} W_{v}^{k} \\
B_{\mu \nu} & =\partial_{\nu} B_{\mu}-\partial_{\mu} B_{v} .
\end{aligned}
$$

A mass term for the $W$ and $B$ gauge bosons would break the $S U(2)_{L} \times U(1)$ gauge symmetry.

Coupled to the gauge fields is a complex scalar $S U(2)_{L}$ doublet, $\Phi$,

$$
\Phi=\left(\begin{array}{c}
\phi^{+} \\
\phi^{0}
\end{array}\right)
$$

with a scalar potential given by

$$
V(\Phi)=\mu^{2}\left|\Phi^{\dagger} \Phi\right|+\lambda\left(\left|\Phi^{\dagger} \Phi\right|\right)^{2},
$$

$(\lambda>0)$. This is the most general renormalizable and $S U(2)_{L}$ invariant potential.

The state of minimum energy for $\mu^{2}<0$ is not at $\phi^{0}=0$ and hence the scalar field develops a vacuum expectation value (VEV). The direction of the minimum in $S U(2)_{L}$ space is not determined since the potential depends only on the combination $\Phi^{\dagger} \Phi$ and we arbitrarily choose

$$
\langle\Phi\rangle=\frac{1}{\sqrt{2}}\left(\begin{array}{l}
0 \\
v
\end{array}\right) .
$$

With this choice the scalar doublet has $U(1)_{Y}$ charge (hypercharge) $Y_{\Phi}=1$ and the electromagnetic charge is ${ }^{2}$

$$
Q_{e m}=\frac{\left(\tau_{3}+Y\right)}{2}
$$

yielding an unbroken electromagnetic charge symmetry:

$$
Q_{e m}\langle\Phi\rangle=0
$$

The contribution of the scalar doublet to the Lagrangian is,

$$
\mathscr{L}_{s}=\left(D^{\mu} \Phi\right)^{\dagger}\left(D_{\mu} \Phi\right)-V(\Phi)
$$

where

$$
D_{\mu}=\partial_{\mu}-i \frac{g}{2} \tau \cdot W_{\mu}-i \frac{g^{\prime}}{2} B_{\mu} Y .
$$

In unitary gauge, the scalar doublet can be written in terms of a physical scalar Higgs field, $h$, as

$$
\Phi=\frac{1}{\sqrt{2}}\left(\begin{array}{c}
0 \\
v+h
\end{array}\right)
$$

\footnotetext{
${ }^{2}$ The $\tau_{i}$ are the Pauli matrices with $\operatorname{Tr}\left(\tau_{i} \tau_{j}\right)=2 \delta_{i j}$.
} 
TABLE 1. Standard Model Particles

\begin{tabular}{lccr}
\hline Field & $S U(3)$ & $S U(2)_{L}$ & $U(1)_{Y}$ \\
\hline$Q_{L}=\left(\begin{array}{c}u_{L} \\
d_{L}\end{array}\right)$ & 3 & 2 & $\frac{1}{3}$ \\
$u_{R}$ & 3 & 1 & $\frac{4}{3}$ \\
$d_{R}$ & 3 & 1 & $-\frac{2}{3}$ \\
$L_{L}=\left(\begin{array}{c}v_{L} \\
e_{L}\end{array}\right)$ & 1 & 2 & -1 \\
$e_{R}$ & 1 & 1 & -2 \\
$\Phi=\left(\begin{array}{c}\phi^{+} \\
\phi^{0}\end{array}\right)$ & 1 & 2 & 1 \\
\hline
\end{tabular}

which gives the contribution to the gauge boson masses from the scalar kinetic energy term of Eq. 8,

$$
M_{\text {Gauge Boson }}^{2} \sim \frac{1}{2}(0, v)\left(\frac{1}{2} g \tau \cdot W_{\mu}+\frac{1}{2} g^{\prime} B_{\mu}\right)^{2}\left(\begin{array}{l}
0 \\
v
\end{array}\right)
$$

The physical gauge fields are two charged fields, $W^{ \pm}$, and two neutral gauge bosons, $Z$ and $A$,

$$
\begin{aligned}
W_{\mu}^{ \pm} & =\frac{1}{\sqrt{2}}\left(W_{\mu}^{1} \mp i W_{\mu}^{2}\right) \\
Z_{\mu} & =\frac{-g^{\prime} B_{\mu}+g W_{\mu}^{3}}{\sqrt{g^{2}+g^{\prime 2}}} \\
A_{\mu} & =\frac{g B_{\mu}+g^{\prime} W_{\mu}^{3}}{\sqrt{g^{2}+g^{\prime 2}}}
\end{aligned}
$$

The gauge bosons obtain masses via Eq. 11:

$$
\begin{aligned}
M_{W}^{2} & =\frac{1}{4} g^{2} v^{2} \\
M_{Z}^{2} & =\frac{1}{4}\left(g^{2}+g^{\prime 2}\right) v^{2} \\
M_{A} & =0 .
\end{aligned}
$$

Three of the degrees of freedom of the complex scalar doublet have been absorbed by the gauge bosons to generate longitudinal polarizations for the $W$ and $Z$ gauge bosons. This is the Higgs mechanism.

Since the massless photon must couple with electromagnetic strength, $e$, the coupling constants define a weak mixing angle $\theta_{W}$,

$$
\begin{aligned}
& e=g \sin \theta_{W} \\
& e=g^{\prime} \cos \theta_{W} .
\end{aligned}
$$




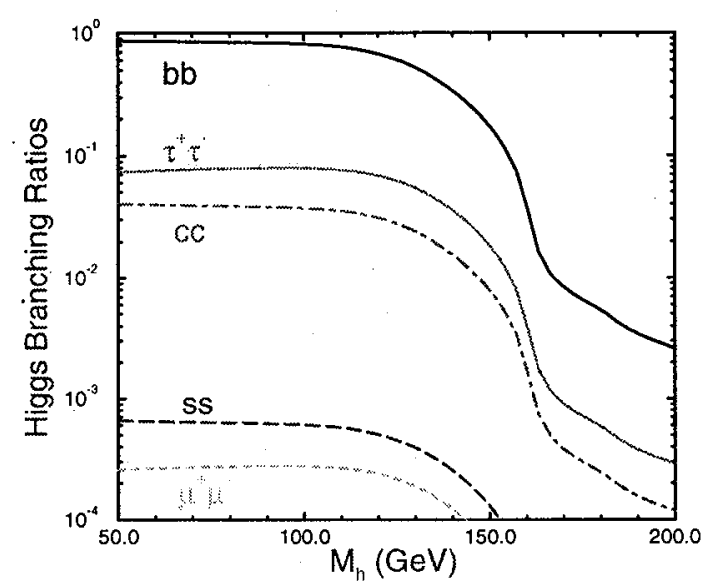

FIGURE 1. Standard Model Higgs boson branching ratios into fermion/anti-fermion pairs.

Fermions can easily be included in the theory and we consider the electron and its neutrino as an example. It is convenient to write the fermions in terms of their left- and right-handed projections,

$$
\psi_{L, R}=\frac{1}{2}\left(1 \mp \gamma_{5}\right) \psi
$$

The lef-handed fermions are assumed to transform as an $S U(2)_{L}$ doublet,

$$
L_{L}=\left(\begin{array}{c}
v_{L} \\
e_{L}
\end{array}\right) \text {. }
$$

From Eq. 6, the hypercharge of the lepton doublet must be $Y_{L}=-1$. Since the neutrino is (at least approximately) massless, it can have only one helicity state which is taken to be $v_{L}$. From the four-Fermi theory of weak interactions, we know that the $W$-boson couples only to left-handed fermions (see for example, Ref. [6]). Experimentally, the righthanded fields do not interact with the $W$ boson, and so the right-handed electron, $e_{R}$, must be an $S U(2)_{L}$ singlet and so has $Y_{e_{R}}=-2$. Using these hypercharge assignments, the leptons can be coupled in a gauge invariant manner to the $S U(2)_{L} \times U(1)_{Y}$ gauge fields,

$$
\mathscr{L}_{\text {lepton }}=i \bar{e}_{R} \gamma^{\mu}\left(\dot{\partial}_{\mu}-i \frac{g^{\prime}}{2} Y_{e} B_{\mu}\right) e_{R}+i \bar{L}_{L} \gamma^{\mu}\left(\partial_{\mu}-i \frac{g}{2} \tau \cdot W_{\mu}-i \frac{g^{\prime}}{2} Y_{L} B_{\mu}\right) L_{L}
$$

All of the known fermions can be accommodated in the Standard Model in a similar manner. The $S U(2)_{L}$ and $U(1)_{Y}$ charge assignments of the first generation of fermions are given in Table 1 . The left-handed fermions are $S U(2)_{L}$ doublets, while the righthanded fermions are $S U(2)_{L}$ singlets. The $S U(3)$ color charge assignments are also listed for convenience.

The parameter $v$ can be found from the charged current for $\mu$ decay, $\mu \rightarrow e \bar{v}_{e} v_{\mu}$, by making the identification,

$$
\frac{G_{\mu}}{\sqrt{2}}=\frac{g^{2}}{8 M_{W}^{2}} .
$$




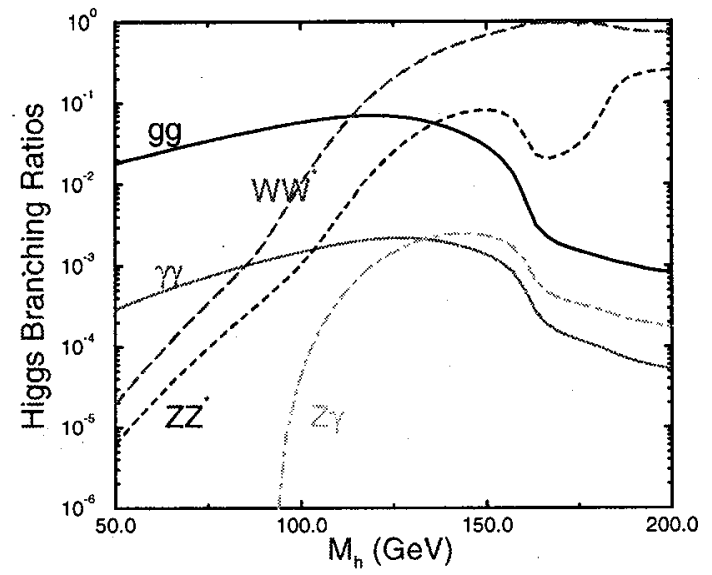

FIGURE 2. Standard Model Higgs boson branching ratios into gauge boson pairs.

The interaction strength for muon decay is measured very accurately to be $G_{\mu}=$ $1.16637 \times 10^{-5} \mathrm{GeV}^{-2}$ and can be used to determine $v=\left(\sqrt{2} G_{\mu}\right)^{-1 / 2}=246 \mathrm{GeV}$.

A fermion mass term takes the form

$$
\mathscr{L}_{\text {mass }}=-m \bar{\psi} \psi=-m\left(\bar{\psi}_{L} \psi_{R}+\bar{\psi}_{R} \psi_{L}\right)
$$

As is obvious from Table 1, the left-and right-handed fermions transform differently under $S U(2)_{L}$ and $U(1)_{Y}$ and electroweak gauge invariance therefore forbids a term of the form of Eq. 19. The Higgs boson can be used to give the fermions mass, however. The gauge invariant Yukawa coupling of the Higgs boson to the up and down quarks is

$$
\mathscr{L}_{d}=-\lambda_{d} \bar{Q}_{L} \Phi d_{R}+\text { h.c. }
$$

This gives the effective coupling,

$$
-\lambda_{d} \frac{1}{\sqrt{2}}\left(\bar{u}_{L}, \bar{d}_{L}\right)\left(\begin{array}{c}
0 \\
v+h
\end{array}\right) d_{R}+\text { h.c. }
$$

which can be seen to yield a mass term for the down quark if we make the identification

$$
\lambda_{d}=\frac{m_{d} \sqrt{2}}{v} .
$$

In order to generate a mass term for the up quark note that $\Phi^{c} \equiv-i \tau_{2} \Phi^{*}$ is an $S U(2)_{L}$ invariant, allowing the coupling,

$$
\mathscr{L}_{u}=-\lambda_{u} \bar{Q}_{L} \Phi^{c} u_{R}+\text { h.c. },
$$

which generates a mass term for the up quark. Similar couplings can be used to generate mass terms for the charged leptons. Since the neutrino has no right handed partner, it 
remains massless. Hence a single scalar Higgs doublet not only generates masses for the gauge bosons, but also for fermions. Unfortunately, the size of the fermion masses remains unexplained.

The couplings of the Higgs boson to the fermions and gauge bosons are directly proportional to their masses (by construction), which has the implication that the Higgs boson decays primarily to the heaviest particles kinematically allowed. At tree level, the Higgs couplings to photons and gluons vanish since the photon and gluon are massless. These couplings first arise at 1-loop and hence are sensitive to new non-SM particles which may propagate in the loops. The Higgs boson branching ratios are shown in Figs. 1 and 2 and they can easily be calculated including higher order corrections using the programs HDECAY[9] or FEYNHIGGS[10].

One of the most important points about the Higgs mechanism is that all of the couplings of the Higgs boson to fermions and gauge bosons are completely determined in terms of gauge coupling constants and fermion masses. The potential of Eq. 4 has two free parameters, $\mu$ and $\lambda$. We can trade these for

$$
\begin{aligned}
v^{2} & =-\frac{\mu^{2}}{2 \lambda}=(246 \mathrm{GeV})^{2} \\
M_{h}^{2} & =2 v^{2} \lambda .
\end{aligned}
$$

There are no remaining adjustable parameters and so Higgs production and decay processes can be computed unambiguously in terms of the Higgs mass alone, making the Higgs sector of the theory completely determined in the SM.

When the scalar potential is expressed in terms of $v$ and $M_{h}$, it becomes,

$$
V=\frac{M_{h}^{2}}{2} h^{2}+\frac{M_{h}^{2}}{2 v} h^{3}+\frac{M_{h}^{2}}{8 v^{2}} h^{4},
$$

and it is apparent that for heavy Higgs masses $\left(M_{h} \sim 1 \mathrm{TeV}\right)$, the Higgs self-interactions become strong.

\section{EXPERIMENTAL SEARCHES FOR THE HIGGS BOSON}

\section{LEP}

The Higgs boson was directly searched for at the LEP collider through the process $e^{+} e^{-} \rightarrow Z h$ at energies up to $\sqrt{s}=209 \mathrm{GeV}$. The Higgs boson decays to the heaviest particles kinematically accessible ( $b \bar{b}$ and $\tau^{+} \tau^{-}$for the LEP searches) and the $Z$ decays roughly $70 \%$ of the time to jets, $20 \%$ to neutrinos, and $10 \%$ to charged leptons. The LEP experiments searched in all of these channels and obtained the limit on a SM Higgs boson[12],

$$
M_{h}>114.4 \mathrm{GeV} \text {. }
$$

This limit can potentially be evaded by constructing models where the Higgs boson decays to non-SM invisible particles with large branching ratios or the Higgs has highly suppressed non-SM couplings to the Z[13]. A Higgs boson with couplings an order of 


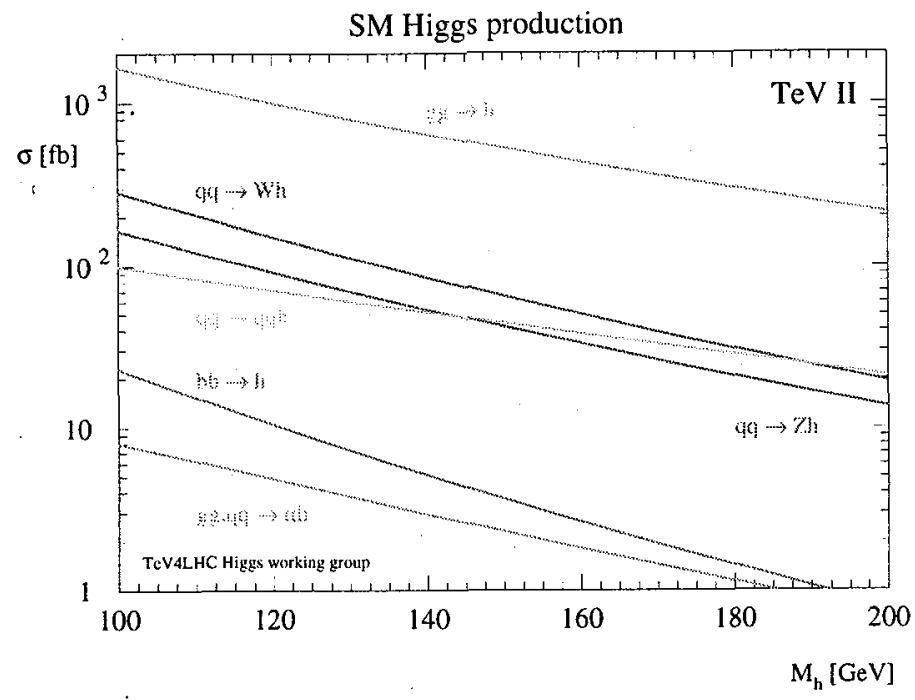

FIGURE 3. Cross sections for SM Higgs boson production processes at the Tevatron $(\sqrt{s}=2 \mathrm{TeV})$, including higher order QCD corrections. From Ref. [11].

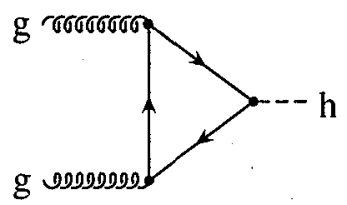

FIGURE 4. Higgs production from gluon fusion. The dominant contribution is from a top quark loop.

magnitude smaller than the SM $h Z Z$ coupling has been ruled out by the LEP experiments for $M_{h}<80 \mathrm{GeV}$.

\section{Tevatron}

The production rates for the SM Higgs boson at the Tevatron are shown in Fig. 3. The largest rate is that for the partonic subprocess $g g \rightarrow h$ (Fig. 4). For $M_{h}<140 \mathrm{GeV}$, the Higgs decays almost entirely to $b \bar{b}$ pairs, as seen from Fig. 1. Unfortunately, the $b \bar{b}$ background is many orders of magnitude larger than the signal and it does not appear possible to extract a Higgs signal from the $g g \rightarrow h \rightarrow b \bar{b}$ channel[5]. For $M_{h}>140 \mathrm{GeV}$, however, the rate to $W W^{* 3}$ grows with increasing Higgs boson mass (see Fig. 2) and becomes large near the $W^{+} W^{-}$threshold. Using this channel, the Tevatron experiments have excluded a single point, $M_{h}=170 \mathrm{GeV}[14]$, as shown in Fig. 5.

The search for a relatively light Higgs boson, $M_{h} \sim 114 \mathrm{GeV}$, proceeds at the Tevatron

${ }^{3} W^{*}$ denotes a virtual $W$ and the branching ratio of Fig. 1 implies a factor of the branching ratio of $W^{*} \rightarrow \bar{f} f^{\prime}$. 


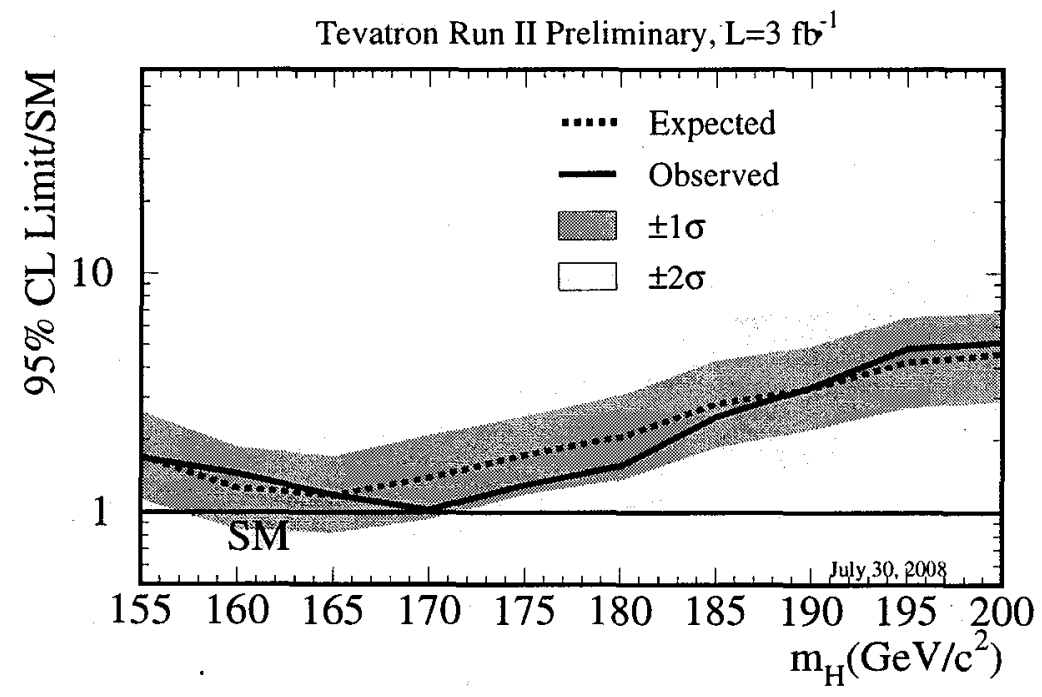

FIGURE 5. Tevatron exclusion of a Higgs boson with $M_{h}=170 \mathrm{GeV}$. From Ref. [14].

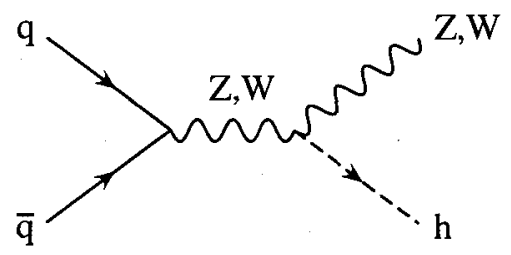

FIGURE 6. Higgs production in association with a vector boson.

primarily through the associated production channel shown in Fig. 6. Although the cross section is smaller than in the gluon fusion channel, the decay products of the $Z$ or $W$ can be tagged and used to reduce the background. The Tevatron limits are normalized to the SM expectations and for $114 \mathrm{GeV}<M_{h}<170 \mathrm{GeV}$ are between a factor of 3-7 above the SM predictions as can be seen in Fig. 7[15]. It is interesting to note that more than 70 different channels are used to obtain Fig. 7, making the statistical combination of individual limits quite complicated. The Tevatron exclusion results are rate limited and are expected to improve with increasing luminosity.

\section{LIMITS FROM PRECISION MEASUREMENTS}

The electroweak sector of the SM can be tested at the multi-loop level due to its predictive nature. In the electroweak sector of the SM, the gauge sector has four fundamental parameters, the $S U(2)_{L} \times U(1)_{Y}$ gauge coupling constants, $g$ and $g^{\prime}$, as well as the two 


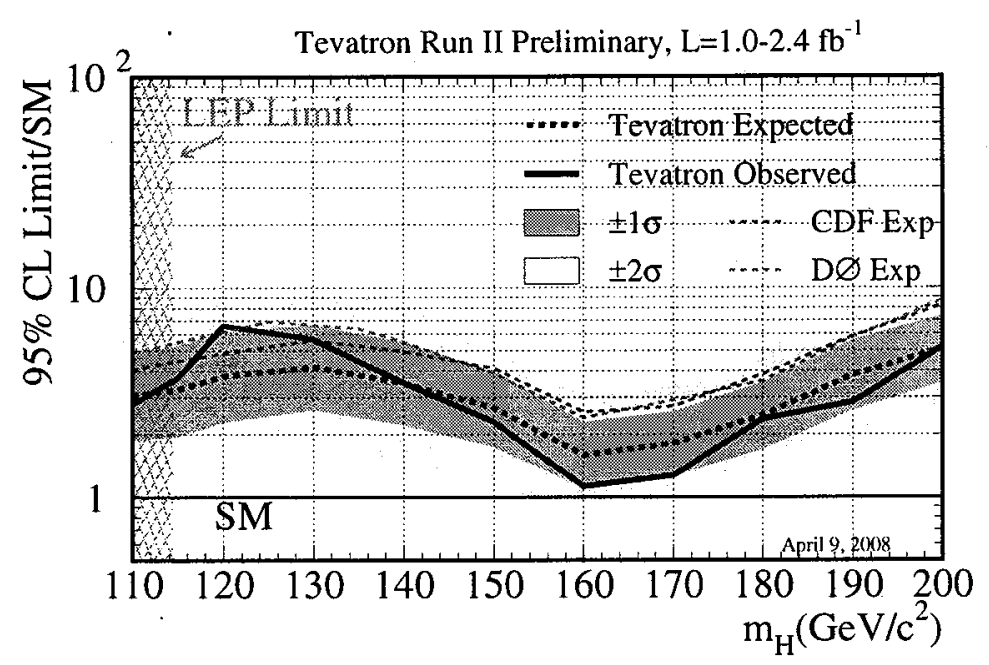

FIGURE 7. $95 \%$ exclusion limit for a SM Higgs boson from the Tevatron experiments. From Ref. [15].

parameters of the Higgs potential, which are usually taken to be the vacuum expectation value of the Higgs boson, $v$, and the Higgs mass, $M_{h}$. Once these parameters are fixed, all other physical quantities in the electroweak sector can be derived in terms of them (and of course the fermion masses and CKM mixing parameters, along with the strong coupling constant $\alpha_{s}$ ). Equivalently, the muon decay constant, $G_{\mu}$, the Z-boson mass, $M_{Z}$, and the fine structure constant, $\alpha$, can be used as input parameters. Experimentally, the measured values for these input parameters are[17],

$$
\begin{aligned}
G_{\mu} & =1.16637(1) \times 10^{-5} \mathrm{GeV}^{-2} \\
M_{Z} & =91.1876(21) \mathrm{GeV} \\
\alpha^{-1} & =137.035999679(94) .
\end{aligned}
$$

The $W$ boson mass is defined through muon decay,

$$
M_{W}^{2}=\frac{\pi \alpha}{\sqrt{2} G_{\mu} s_{\theta}^{2}} .
$$

The SM satisfies $\rho=1$ at tree level and predicts the weak mixing angle in terms of the gauge boson masses,

$$
\rho=1=\frac{M_{W}^{2}}{M_{Z}^{2} c_{\theta}^{2}} .
$$

At tree level, all definitions of the weak mixing angle (Eq. 14) are equivalent, but the definitions differ at higher order. In Eq. 31, $M_{W}$ and $M_{Z}$ are the physical gauge boson masses, and this definition of the weak mixing angle, $s_{\theta}$, corresponds to the on-shell 


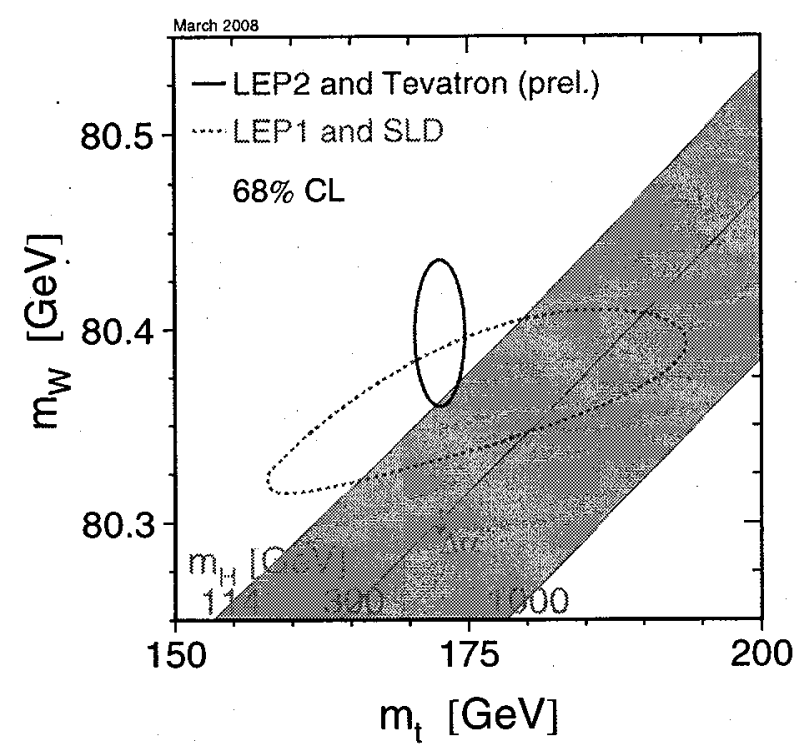

FIGURE 8. Comparison of the direct search results from LEP2 and the Tevatron for $M_{w}$ and $m_{t}$ with those inferred from the consistency of the SM. From Ref. [16].

scheme. Eqs. 30 and $3 i$ imply,

$$
M_{W}^{2}=\frac{M_{Z}^{2}}{2}\left\{1+\sqrt{1-\frac{4 \pi \alpha}{\sqrt{2} G_{\mu} M_{Z}^{2}}}\right\} .
$$

At tree level, the SM therefore predicts from Eq. 32,

$$
M_{W}(\text { tree })=79.829 \mathrm{GeV},
$$

in disagreement with the measured value[16],

$$
M_{W}(\text { experiment })=80.399 \pm 0.025 \mathrm{GeV} .
$$

In order to obtain good agreement between theory and the experimental data, it is crucial to include radiative corrections[18, 19]. For example, the prediction for $M_{W}$ can be expressed as[20],

$$
M_{W}^{2}=\frac{\pi \alpha}{\sqrt{2} G_{\mu} s_{\theta}^{2}}\left[1+\Delta r_{S M}\right]
$$

where $\Delta r_{S M}$ summarizes the radiative corrections. The dependence on the top quark mass, $m_{t}$, is particularly significant as $\Delta r_{S M}$ depends on $m_{t}$ quadratically,

$$
\Delta r_{S M}^{t}=-\frac{G_{\mu}}{\sqrt{2}} \frac{N_{c}}{8 \pi^{2}}\left(\frac{c_{\theta}^{2}}{s_{\theta}^{2}}\right) m_{t}^{2}+\log \left(m_{t}\right) \text { terms }
$$




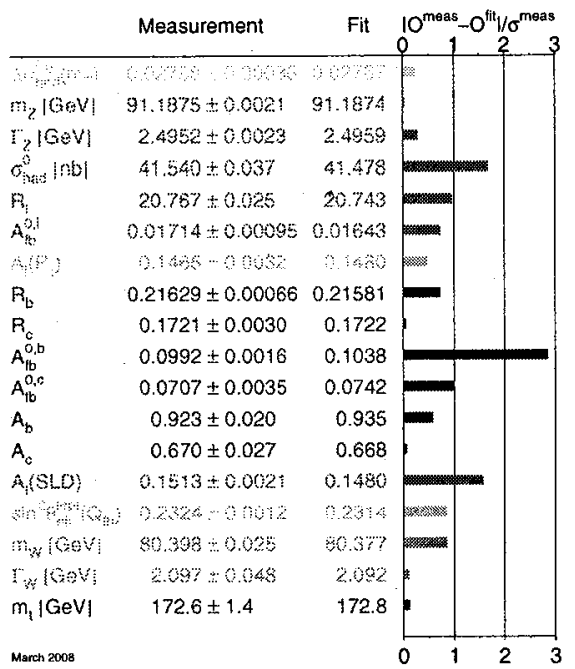

FIGURE 9. Comparison of electroweak measurements with a best fit to the SM theory. From Ref. [16].

where $N_{c}=3$ is the number of colors.

The top quark does not decouple from the theory even at energies far above the top quark mass. The decoupling theorem[21] (which says that heavy particles do not affect low scale physics) is violated by the top quark because the top quark couplings to both the Higgs boson and the longitudinal components of the gauge bosons are proportional to $m_{t}$ and also because the SM is not renormalizable without the top quark.

The dependence of $M_{W}$ and other electroweak observables on the Higgs boson mass is logarithmic and so predictions are much less sensitive to $M_{h}$ than to $m_{t}$. The complete contribution to $\Delta r_{S M}$ can be approximated for a heavy Higgs[20],

$$
\Delta r_{S M}=.070+\Delta r_{S M}^{t}+\frac{\alpha}{\pi s_{\theta}^{2}} \frac{11}{48}\left[\log \left(\frac{M_{h}^{2}}{M_{Z}^{2}}\right)-\frac{5}{6}\right]+2 \text {-loop }
$$

The first term in Eq. 37 results primarily from the scaling of $\delta \alpha$ from $q^{2}=0$ to $M_{Z}$.

The agreement between the prediction for the $W$ mass given by Eqs. 35 and 37 with the measured value is a strong test of the theory. The measurements of $M_{W}$ and $m_{t}$ can be used to infer limits on the Higgs boson mass, as can be seen in Fig. 8. A relatively light value of $M_{h}$ is clearly prefered. The measured values for some high energy observables from LEP, SLC, and the Tevatron are listed in the left hand column of Fig. 9. The best fit to the predictions of the SM (including radiative corrections) is given in the right hand column. The agreement between the data and the predictions is compelling evidence for the validity of the SM at current energy scales. From Fig. 8, we see that the measured $W$ mass is slightly high compared with the value extracted from the precision electroweak data of Fig. 9. 


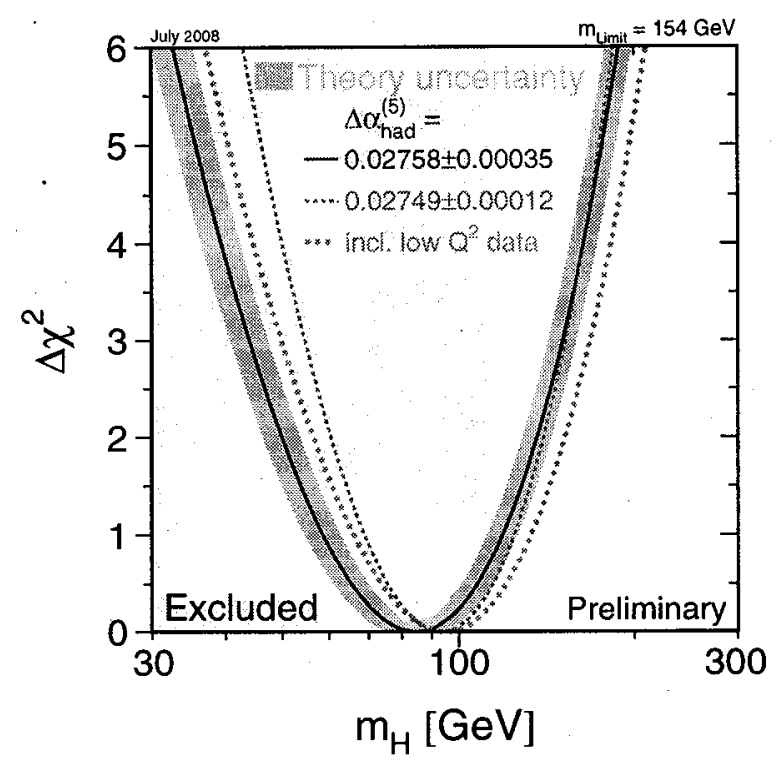

FIGURE 10. Best fit to the Higgs boson mass from data at LEP, SLC, and the Tevatron. From Ref. [16].

The data can be used to obtain a prediction for the Higgs boson mass as seen in the "blue-band" plot of Fig. 10. When the direct Higgs search results from LEP and the Tevatron and the results from low energy experiments such as atomic parity violation are omitted, the best fit is[16],

$$
M_{h}=84_{-26}^{+34} \mathrm{GeV} .
$$

It is somewhat distressing that the best fit from the observables of Fig. 9 is in the region excluded by the LEP direct search, Eq. 26. When the direct search results from LEP are included, a $95 \%$ confidence level upper bound is found,

$$
M_{h}<185 \mathrm{GeV} .
$$

This limit assumes that there are no particles which contribute to the radiative corrections to the SM observables of Fig. 9 beyond the SM particles (and also that all couplings have their SM values). It is quite easy to evade the limit of Eq. 39 in extentions of the SM.

The fits shown in Figs. 8, 9 and 10 were performed by the LEP Electroweak Working Group. Other groups have also done global fits with slightly different methodologies and assumptions $[22,23]$. The GFITTER collaboration has a version of the blue-band plot shown in Fig. 11 which includes theory uncertainties, which can be seen to be significant. The best fit to the Higgs mass from the GFITTER collaboration is,

$$
M_{h}=80_{-23}^{+30} \mathrm{GeV}
$$

in good agreement with the LEP Electroweak Working Group fit of Eq. 38. The GFITTER result including the direct search results from both LEP and from $3 \mathrm{fb}^{-1}$ of data at 


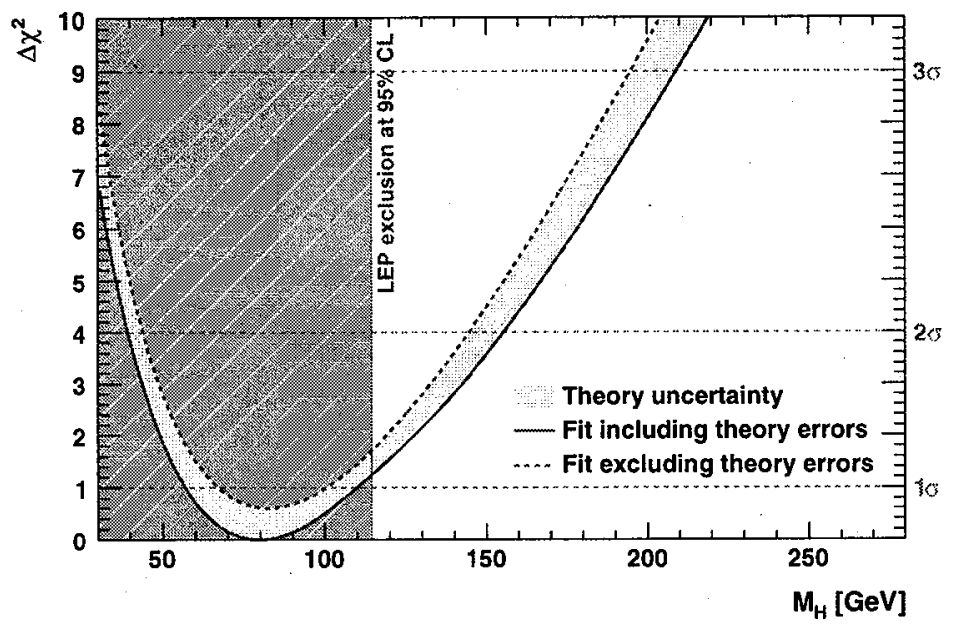

FIGURE 11. Blue-band plot from the GFITTER collaboration including an estimate of theory errors. From Ref. [22].

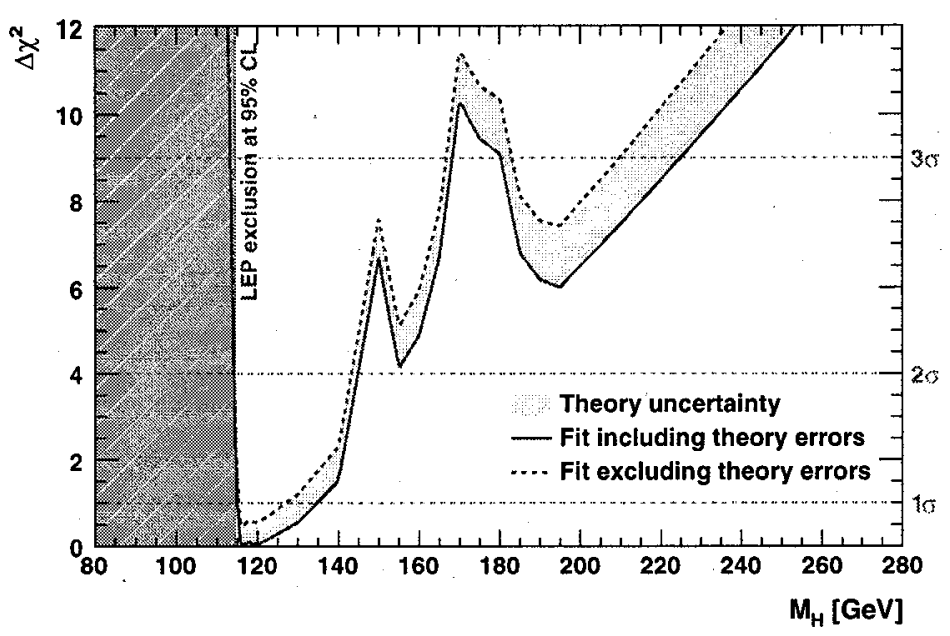

FIGURE 12. Blue-band plot from the GFITTER collaboration including the direct search limits from LEP and from the Tevatron experiments with $3 \mathrm{fb}^{-1}$ of data[22].

the Tevatron (Figs. 5 and 7) is shown in Fig. 12. It is interesting that the Tevatron search results are beginning to influence the global fit to the Higgs mass.

\section{WHY IS THE STANDARD MODEL UNSATISFACTORY?}

Although the SM is for the most part consistent with experimental data, most theorists believe that it is incomplete. In this section, I summarize the arguments for the existence of physics beyond the SM. 


\section{Perturbativity and Triviality}

Theoretical bounds on the Higgs boson mass have been deduced on the grounds of triviality, $[24,25,26]$ which can be summarized as the requirement that the Higgs quartic coupling remain finite at high energy scales. Consider the scalar sector of the SM, ${ }^{4}$

$$
V(\Phi)=\mu^{2}\left|\Phi^{\dagger} \Phi\right|+\lambda\left(\left|\Phi^{\dagger} \Phi\right|\right)^{2}
$$

where the quartic coupling is

$$
\lambda=\frac{M_{h}^{2}}{2 v^{2}} .
$$

The quartic coupling, $\lambda$, changes with the effective energy scale $Q$ due to the self interactions of the scalar field:

$$
\frac{d \lambda}{d t}=\frac{3 \lambda^{2}}{4 \pi^{2}}
$$

where $t \equiv \log \left(Q^{2} / Q_{0}^{2}\right)$ and $Q_{0}$ is some reference scale. Solving Eq. 43,

$$
\frac{1}{\lambda(Q)}=\frac{1}{\lambda\left(Q_{0}\right)}-\frac{3}{4 \pi^{2}} \log \left(\frac{Q^{2}}{Q_{0}^{2}}\right)
$$

Summing the geometric series,

$$
\lambda(Q)=\frac{\lambda\left(Q_{0}\right)}{\left[1-\frac{3 \lambda\left(Q_{0}\right)}{4 \pi^{2}} \log \left(\frac{Q^{2}}{Q_{0}^{2}}\right)\right]} .
$$

From Eq. 45 we see that $\lambda(Q)$ blows up as $Q \rightarrow \infty$ (called the Landau pole). Regardless of how small $\lambda\left(Q_{0}\right)$ is, $\lambda(Q)$ will become infinite at some large value of $Q$. Alternatively, $\lambda\left(Q_{0}\right) \rightarrow 0$ as $Q \rightarrow 0$ with $\lambda(Q)>0$.

The requirement that the quartic coupling be finite at a high scale $\Lambda$,

$$
\frac{1}{\lambda(\Lambda)}>0
$$

can be interpreteted as a bound on the Higgs boson mass,

$$
M_{h}^{2}<\frac{8 \pi^{2} v^{2}}{3 \log \left(\Lambda^{2} / v^{2}\right)},
$$

(where we set $Q_{0}=v$ ). Requiring that the SM be valid up to the scale associated with grand unified models, $\Lambda \sim 10^{16} \mathrm{GeV}$, yields the approximate upper bound,

$$
M_{h}<160 \mathrm{GeV} \text {. }
$$

${ }^{4} \mu^{2}<0, \lambda>0$ 


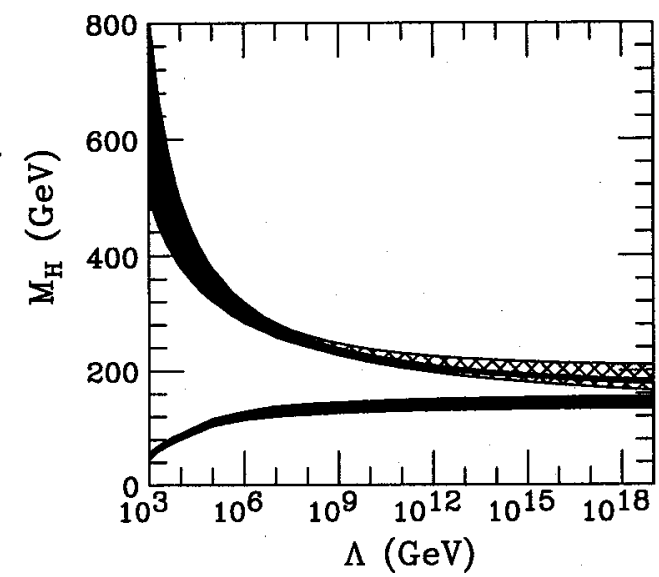

FIGURE 13. Theoretical limits on the Higgs boson mass as a function of the scale of new physics, $\Lambda$. The allowed region is between the curves. From Ref. [26].

As the scale $\Lambda$ becomes smaller, the limit on the Higgs mass becomes progressively weaker. For large $\Lambda$, of course, higher order and non-perturbative corrections must be included[27]. $\Lambda$ is often interpreted as the scale of new physics, since above the scale $\Lambda$ the $\mathrm{SM}$ is no longer a sensible theory.

Another bound on the Higgs mass can be derived by the requirement that spontaneous symmetry breaking occur,

$$
V(v)<V(0) \text {. }
$$

This bound is essentially equivalent to the requirement that $\lambda$ remain positive at all scales $\Lambda$. (If $\lambda$ becomes negative, the potential is unbounded from below and has no state of minimum energy.) For small $\lambda$, the scaling is [3],

$$
\frac{d \lambda}{d t}=\frac{1}{16 \pi^{2}}\left[-12 g_{t}^{4}+\frac{3}{16}\left(2 g^{4}+\left(g^{2}+g^{\prime 2}\right)^{2}\right)\right],
$$

where $g_{t}=m_{t} / v$ is the top quark Yukawa coupling. Eq. 50 is easily solved to find,

$$
\lambda(\Lambda)=\lambda(v)+\frac{1}{16 \pi^{2}}\left[-12 g_{t}^{4}+\frac{3}{16}\left(2 g^{4}+\left(g^{2}+g^{\prime 2}\right)^{2}\right)\right] \log \left(\frac{\Lambda^{2}}{v^{2}}\right) .
$$

Requiring $\lambda(\Lambda)>0$ gives the bound on the Higgs boson mass,

$$
M_{h}^{2}>\frac{v^{2}}{8 \pi^{2}}\left[-12 g_{t}^{4}+\frac{3}{16}\left(2 g^{4}+\left(g^{2}+g^{2}\right)^{2}\right)\right] \log \left(\frac{\Lambda^{2}}{v^{2}}\right) .
$$

A more careful analysis along the same lines as above [27] using the 2 loop renormalization group improved effective potential ${ }^{5}$ and the running of all couplings gives the

\footnotetext{
5 The renormalization group improved effective potential sums all potentially large logarithms, $\log \left(Q^{2} / v^{2}\right)$.
} 


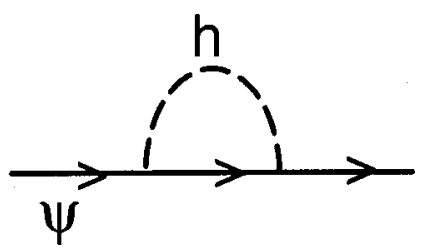

FIGURE 14. Fermion mass renormalization from an internal Higgs boson.

requirement that if the Standard Model is valid up to scales of order $10^{16} \mathrm{GeV}$, then [27]

$$
M_{h}(\mathrm{GeV})>130+2\left(m_{t}-170\right) .
$$

Eqs. 47 and 53 imply that if the SM is valid up to around $10^{16} \mathrm{GeV}$, then the Higgs mass is restricted to be between approximately $126 \mathrm{GeV}$ and $160 \mathrm{GeV}[26,27,28,29]$. It is interesting that this is precisely the region preferred by the electroweak precision obervables of the previous section. As the scale $\Lambda$ is reduced, the allowed range for the Higgs mass is enlarged. The theoretically allowed region for the Higgs mass as a function of the scale $\Lambda$ is shown in Fig. 13. It is important to remember that this bound assumes the SM with a single Higgs doublet. In extentions of the SM with extra Higgs doublets (for example, in supersymmetric models), it is possible to evade the bound of Eq. 53.

\section{Naturalness}

One of the most glaring theoretical inadequacies of the SM arises when we compute quantum corrections to the Higgs boson mass. One-loop corrections to the Higgs mass have the undesirable feature that they depend quadratically on high scale physics [30]. The basic point can be illustrated with a simplified version of the SM containing a single fermion, $\psi$, coupled to a massive Higgs scalar, $\phi$,

$$
\mathscr{L}_{\phi}=\bar{\psi}(i \partial) \psi+\left|\partial_{\mu} \phi\right|^{2}-m_{S}^{2}|\phi|^{2}-\left(\frac{\lambda_{F}}{2} \bar{\psi} \psi \phi+\text { h.c. }\right) .
$$

Assume that this Lagrangian leads to spontaneous symmetry breaking and $\phi=(h+$ $v) / \sqrt{2}$, with $h$ a physical Higgs boson. After spontaneous symmetry breaking, the fermion acquires a mass, $m_{F}=\lambda_{F} v / \sqrt{2}$. Consider the fermion self-energy arising from the scalar loop corresponding to Fig. 14.

$$
-i \Sigma_{F}(p)=\left(\frac{-i \lambda_{F}}{\sqrt{2}}\right)^{2}(i)^{2} \int \frac{d^{4} k}{(2 \pi)^{4}} \frac{\left(k+m_{F}\right)}{\left[k^{2}-m_{F}^{2}\right]\left[(k-p)^{2}-m_{S}^{2}\right]} .
$$

The renormalized fermion mass is $m_{F}^{r}=m_{F}+\delta m_{F}$, with

$$
\begin{aligned}
\delta m_{F} & =\left.\Sigma_{F}(p)\right|_{p=m_{F}} \\
& =i \frac{\lambda_{F}^{2}}{32 \pi^{4}} \int_{0}^{1} d x \int d^{4} k^{\prime} \frac{m_{F}(1+x)}{\left[k^{2}-m_{F}^{2} x^{2}-m_{S}^{2}(1-x)\right]^{2}} .
\end{aligned}
$$




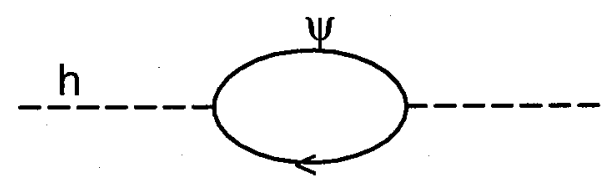

FIGURE 15. Fermion mass renormalization from a fermion loop, $\phi$.

The integral can be performed in Euclidean space with a momentum space cut-off using the fact that for a symmetric integral[31],

$$
\int d^{4} k_{E} f\left(k_{E}^{2}\right)=\pi^{2} \int_{0}^{\Lambda^{2}} y d y f(y)
$$

In Eq. 57, $\Lambda$ is a high energy cut-off, presumably of the order of the Planck scale or a grand unified scale. The renormalization of the fermion mass is,

$$
\begin{aligned}
\delta m_{F} & =-\frac{\lambda_{F}^{2} m_{F}}{32 \pi^{2}} \int_{0}^{1} d x(1+x) \int_{0}^{\Lambda^{2}} \frac{y d y}{\left[y+m_{F}^{2} x^{2}+m_{S}^{2}(1-x)\right]^{2}} \\
& =-\frac{3 \lambda_{F}^{2} m_{F}}{64 \pi^{2}} \log \left(\frac{\Lambda^{2}}{m_{F}^{2}}\right)+\ldots
\end{aligned}
$$

where the .... indicates terms independent of the cutoff or which vanish when $\Lambda \rightarrow \infty$. This correction clearly corresponds to a well-defined expansion for $m_{F}$.

In the limit in which the fermion mass vanishes, Eq. 54 is invariant under the chiral transformations,

$$
\begin{aligned}
& \psi_{L} \rightarrow e^{i \theta_{L}} \psi_{L} \\
& \psi_{R} \rightarrow e^{i \theta_{R}} \psi_{R},
\end{aligned}
$$

and setting the fermion mass to zero increases the symmetry of the theory. Since the Yukawa coupling (proportional to the fermion mass term) breaks this symmetry, the corrections to the mass must be proportional to $m_{F}$.

The situation is quite different, however, for the renormalization of the scalar mass from a fermion loop (Fig. 15) using the same Lagrangian (Eq. 54),

$$
-i \Sigma_{S}\left(p^{2}\right)=\left(\frac{-i \lambda_{F}}{\sqrt{2}}\right)^{2}(i)^{2}(-1) \int \frac{d^{4} k}{(2 \pi)^{4}} \frac{\operatorname{Tr}\left[\left(k+m_{F}\right)\left((k-p)+m_{F}\right)\right]}{\left(k^{2}-m_{F}^{2}\right)\left[(k-p)^{2}-m_{F}^{2}\right]} .
$$

Integrating as before with a momentum space cutoff,

$$
\delta M_{h}^{2}=-\frac{\lambda_{F}^{2}}{8 \pi^{2}} \Lambda^{2}+\ldots
$$

The Higgs boson mass depends quadratically on the high scale cut-off $\Lambda$. Note that the correction is not proportional to $M_{h}$. Setting the Higgs mass equal to zero does not 
increase the symmetry of the Lagrangian and there is nothing that protects the Higgs mass from large corrections.

In the Standard Model, we expect that the physical Higgs boson mass, $M_{h}$, is of the order of a few hundred $\mathrm{GeV}$ from the precision results discussed in the previous section. The quadratic contributions to the Higgs boson mass renormalization in the SM are[29],

$$
\begin{aligned}
\delta M_{h}^{2} & =\frac{3}{8 \pi v^{2}} \Lambda^{2}\left(6 M_{W}^{2}+3 M_{Z}^{2}+3 M_{h}^{2}-12 M_{t}^{2}\right) \\
& \sim-\left(\frac{\Lambda}{.7 \mathrm{TeV}} 200 \mathrm{GeV}\right)^{2} .
\end{aligned}
$$

Eq. 62 suggests that in order not to have large cancellations, $\Lambda$ should be $\mathscr{O}(\mathrm{TeV})$. This is known as the hierarchy problem: Why should $\Lambda$ be $\mathscr{O}(\mathrm{TeV})$ and not the Planck scale? Understanding the hierarchy problem as expressed by Eq. 62 has stimulated much model building. The basic approach is to postulate new particles which contribute to the Higgs mass renormalization at one loop and cancel the SM contributions. Supersymmetric models do this by postulating scalar particles associated with the known fermions with just the right couplings to cancel the SM contributions to Eq. 62, while Little Higgs type models cancel the SM quadratic contributions using particles with the same spin as the SM particles. In both cases, the models contain $\mathrm{TeV}$ scale particles which can potentially be observed at the LHC.

\section{Unitarity}

A different type of limit on the SM parameters is obtained by looking at high energy scattering. For a $2 \rightarrow 2$ elastic scattering process, the differential cross section is

$$
\frac{d \sigma}{d \Omega}=\frac{1}{64 \pi^{2} s}|\mathscr{A}|^{2} .
$$

Using a partial wave decomposition, the amplitude can be written as

$$
\mathscr{A}=16 \pi \sum_{l=0}^{\infty}(2 l+1) P_{l}(\cos \theta) a_{l},
$$

where $a_{l}$ is the spin $l$ partial wave and $P_{l}(\cos \theta)$ are the Legendre polynomials. The cross section is,

$$
\begin{aligned}
\sigma & =\frac{8 \pi}{s} \sum_{l=0}^{\infty} \sum_{l^{\prime}=0}^{\infty}(2 l+1)\left(2 l^{\prime}+1\right) a_{l} a_{l}^{*} \int_{-1}^{1} d \cos \theta P_{l}(\cos \theta) P_{l^{\prime}}(\cos \theta) \\
& =\frac{16 \pi}{s} \sum_{l=0}^{\infty}(2 l+1)\left|a_{l}\right|^{2},
\end{aligned}
$$

where we have used the fact that the $P_{l}$ 's are orthogonal. The optical theorem gives,

$$
\sigma=\frac{1}{s} \operatorname{Im}[\mathscr{A}(\theta=0)]=\frac{16 \pi}{s} \sum_{l=0}^{\infty}(2 l+1)\left|a_{l}\right|^{2} .
$$


This immediately yields the unitarity requirement,

$$
\left|a_{l}\right|^{2}=\operatorname{Im}\left(a_{l}\right)
$$

or equivalently,

$$
\left|\operatorname{Re}\left(a_{l}\right)\right|<\frac{1}{2}
$$

As a demonstration of restrictions coming from the requirement of perturbative unitarity, consider the scattering of longitudinal gauge bosons, $W_{L}^{+} W_{L}^{-} \rightarrow W_{L}^{+} W_{L}^{-}$, The $J=0$ partial wave, $a_{0}^{0}$, in the limit $M_{W}^{2}<<s$, is[32, 33],

$$
\begin{aligned}
a_{0}^{0}\left(W_{L}^{+} W_{L}^{-} \rightarrow W_{L}^{+} W_{L}^{-}\right) & \equiv \frac{1}{16 \pi s} \int_{-s}^{0}|\mathscr{A}| d t \\
& =-\frac{M_{h}^{2}}{16 \pi v^{2}}\left[2+\frac{M_{h}^{2}}{s-M_{h}^{2}}-\frac{M_{h}^{2}}{s} \log \left(1+\frac{s}{M_{h}^{2}}\right)\right]
\end{aligned}
$$

At very high energy, $s>>M_{h}^{2}$, Eq. 69 has the limit

$$
a_{0}^{0} \longrightarrow_{s>>M_{h}^{2}}-\frac{M_{h}^{2}}{8 \pi v^{2}} .
$$

Applying the unitarity condition, $\left|\operatorname{Re}\left(a_{0}^{0}\right)\right|<\frac{1}{2}$ gives the restriction,

$$
M_{h}<870 \mathrm{GeV} \text {. }
$$

It is important to understand that this does not mean that the Higgs boson cannot be heavier than $870 \mathrm{GeV}$, it simply means that for heavier Higgs boson masses perturbation theory is not valid. The Higgs boson plays a fundamental role in the theory since it cuts off the growth of the partial wave amplitudes and makes the theory obey perturbative unitarity.

Taking the alternate limit, $s<<M_{h}^{2}$,

$$
a_{0}^{0} \longrightarrow s<<M_{h}^{2}-\frac{s}{32 \pi v^{2}} .
$$

Again applying the unitarity condition, we obtain,

$$
\sqrt{s_{c}}<1.7 \mathrm{TeV}
$$

The notation $s_{c}$ denotes $s$ (critical), the scale at which perturbative unitarity is violated. Eq. 73 is the basis for the oft-repeated statement, There must be new physics on the TeV scale. It is encouraging that Eq. 73 is exactly the energy scale that will be explored at the LHC. 


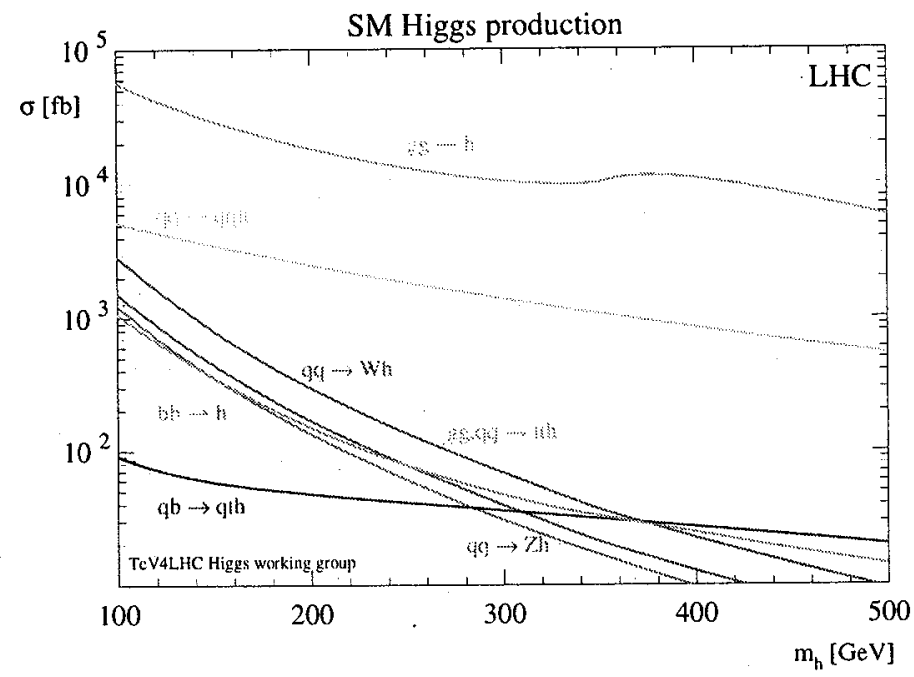

FIGURE 16. Cross sections for SM Higgs boson production processes at the LHC $(\sqrt{s}=14 \mathrm{TeV})$, including higher order corrections. From Ref. [11].

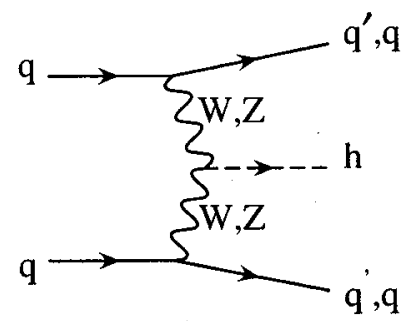

FIGURE 17. Higgs production from vector boson fusion.

\section{SEARCHES FOR THE HIGGS BOSON AT THE LHC}

The LHC is expected to find the Higgs boson for all Higgs masses less than around $800 \mathrm{GeV}[34,35]$. The production cross sections are large (Fig. 16) and the theoretical predictions are well understood, with all important Higgs production channels known to at least next-to-leading order accuracy[36]. As is the case at the Tevatron, the largest production mechanism is gluon fusion, but again the largest decay for light Higgs bosons, $h \rightarrow b \bar{b}$, has an overwhelming QCD background. Above about $M_{h} \sim 140 \mathrm{GeV}$, the Higgs decays to $W W^{*}$ and $Z Z^{*}$ can be used for a Higgs discovery.

The vector boson fusion channel (Fig. 17), which is not important at the Tevatron, is useful for Higgs discovery over a large Higgs mass region at the LHC[5]. By tagging the forward jets associated with the Higgs production, the background can be significantly reduced. This channel can potentially be used to observe the decay $h \rightarrow \tau^{+} \tau^{-}$[37] and $h \rightarrow W^{+} W^{-}[38]$. 


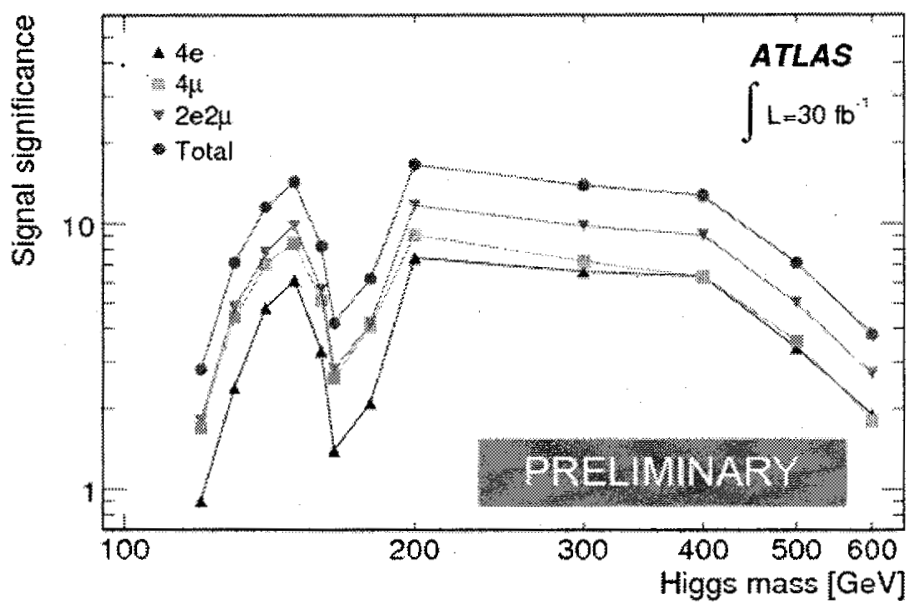

FIGURE 18. Significance of the $h \rightarrow Z Z^{*} \rightarrow 4$ leptons discovery channel using the ATLAS detector at the LHC with $30 \mathrm{fb}^{-1}$. From Ref. [39].

$$
h \rightarrow \gamma \gamma
$$

Although the branching ratio is $\mathscr{O}\left(10^{-3}-10^{-4}\right)$, (see Fig. 2), the Higgs boson can potentially be discovered in the $g g \rightarrow h \rightarrow \gamma \gamma$ channel for lighter Higgs bosons $\left(M_{h}<\right.$ $140 \mathrm{GeV})$. For $M_{h}>140 \mathrm{GeV}$, the event rate becomes too small to be observed. The largest reducible backgrounds are $q \bar{q} \rightarrow \gamma \gamma$ and $g g \rightarrow \gamma \gamma$ which can be directly measured from the sidebands away from the Higgs boson peak. There are also large reducible backgrounds from $\gamma$-jet and jet-jet production where the jet is misidentified as a photon. Excellent $\gamma$-jet separation and $\gamma$ energy resolution help eliminate these backgrounds. Both the ATLAS and CMS collaborations have redone their original analyses to optimize the event selection. CMS finds that a significance of $>8 \sigma$ in the $h \rightarrow \gamma \gamma$ channel can be achieved for $M_{h} \sim 130 \mathrm{GeV}$ with an integrated luminosity of $30 \mathrm{fb}^{-1}$ [39], while the ATLAS studies are less optimistic[39].

$$
h \rightarrow Z Z
$$

For $M_{h}>130 \mathrm{GeV}$, the Higgs boson can be discovered in the so-called golden channel, $h \rightarrow Z Z^{*} \rightarrow 4$ leptons, except for near the $W^{+} W^{-}$threshold. This channel can be used for Higgs masses up to around $M_{h} \sim 600 \mathrm{GeV}$ and has a smooth background and a clean signature with a peak in the 4-lepton invariant mass allowing for complete reconstruction of the Higgs mass. The background can be measured from the sidebands and the estimated sensitivity for $\int L=30 \mathrm{fb}^{-1}$ is shown in Fig. 18. 


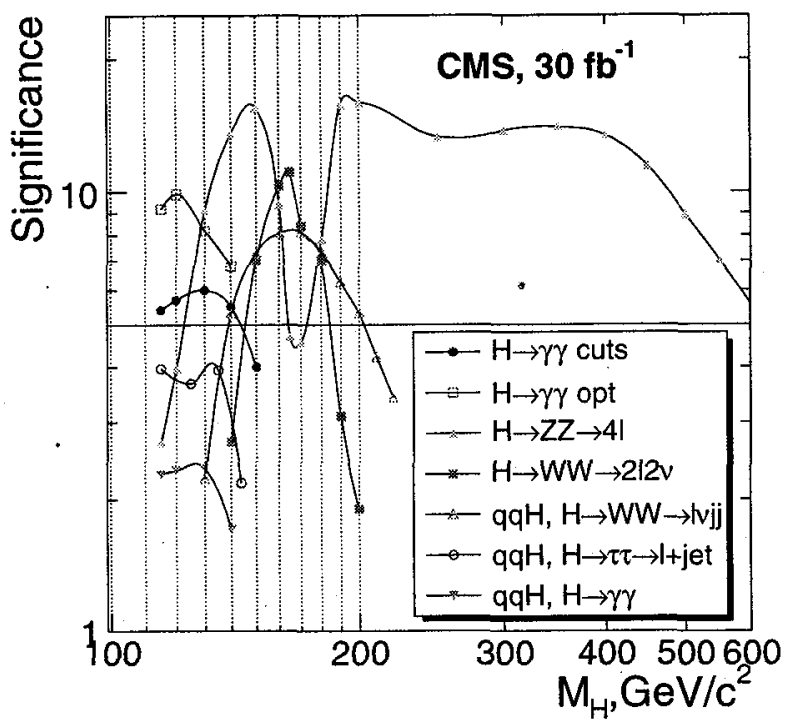

FIGURE 19. Significance of a Higgs discovery at CMS with $30 \mathrm{fb}^{-1}$. From Ref. [35].

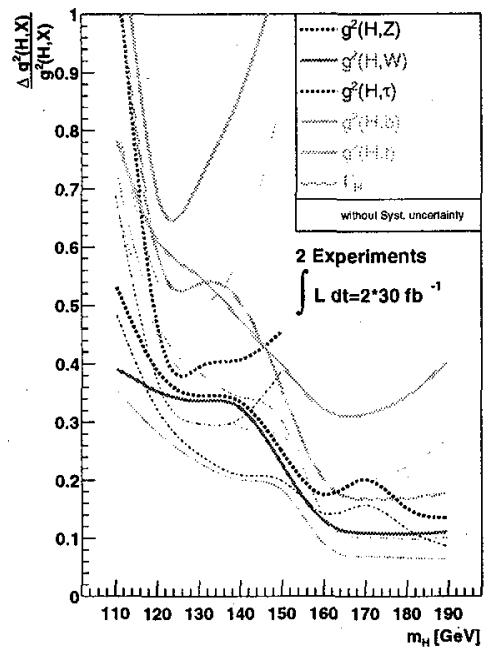

FIGURE 20. Potential sensitivity for Higgs coupling measurements at the LHC. From Ref. [40].

\section{Sensitivity}

The estimated sensitivity for a Higgs discovery from the CMS experiment is shown in Fig. 19 for an integrated luminosity of $\int L=30 \mathrm{fb}^{-1}$. It is important to note that for any given Higgs mass, only a few channels are accessible and for $M_{h}>200 \mathrm{GeV}$ only the $h \rightarrow Z Z \rightarrow 4$ leptons will be accessible at the initial luminosity. The significance is greater than 5 for all values of the Higgs mass with $30 \mathrm{fb}^{-1}$.

After discovering the Higgs boson, the next task is to measure its properties as precisely as possible to see if it is a SM Higgs boson. We need to measure the spin/parity, 
the Higgs couplings to fermions and gauge bosons, and the Higgs self-couplings. All of these measurements will be extremely challenging at the LHC. As an example, we show in Fig. 20 the precision which with the Higgs couplings can potentially be measured at the LHC $[40,41]$.

\section{CONCLUSION}

With the turn on of the LHC, particle physics will enter a new era of electroweak physics. There are three possibilities for the Higgs sector. First, the Higgs could be discovered with SM-like properties and a mass consistent with the electroweak precision observables. In this case, the problem of naturalness has no obvious solution and the only course will be to measure the Higgs properties with great precision. The second possibility is that a Higgs boson is discovered with SM-like properties, but with a mass inconsistent with electroweak precision observables. This case will keep theorists busy building models. Finally, it is possible that no Higgs boson will be discovered. In this case, the problem of unitarity comes to the forefront. In all three cases, it is possible (and quite likely) that new particles outside the Higgs sector will be discovered. Whatever the scenario, we are bound to learn about the electroweak sector!

\section{ACKNOWLEDGMENTS}

Thanks to Maria Elena Tejeda-Yeomans, Alejandro Ayala, and the other organizers for a very enjoyable and interesting meeting. I thank the students for asking questions which made me think! This work was supported by the U.S. Department of Energy under grant DE-AC02-98CH10886.

\section{REFERENCES}

1. A. Djouadi, Phys. Rept. 457, 1 (2008), [hep-ph/0503172].

2. C. Quigg, arXiv:0704.2045 [hep-ph].

3. J. F. Gunion, H. E. Haber, G. L. Kane and S. Dawson, Cambridge, USA: Perseus Publishing (1990).

4. M. E. Peskin and D. V. Schroeder, Reading, USA: Addison-Wesley (1995) 842 p.

5. D. Rainwater, hep-ph/0702124.

6. C. Quigg, Front. Phys. 56, 1 (1983).

7. S. Dawson, hep-ph/9901280.

8. L. Reina, hep-ph/0512377.

9. M. Spira, http://people.web.psi.ch/spira/proglist.html.

10. M. Frank et al., JHEP 02, 047 (2007), [hep-ph/0611326].

11. U. Aglietti et al., hep-ph/0612172.

12. LEP Working Group for Higgs boson searches, R. Barate et al., Phys. Lett. B565, 61 (2003), [hepex/0306033].

13. S. Chang, R. Dermisek, J. F. Gunion and N. Weiner, Ann. Rev. Nucl. Part. Sci. 58, 75 (2008), [0801.4554].

14. C. Herndon, M. for the Babar and D. Collaborations, 0810.3705.

15. CDF, T. T. W. Group, 0804.3423.

16. LEP Electroweak Working Group, http://lepewwg.web.cern.ch/LEPEWWG/.

17. Particle Data Group, C. Amsler et al., Phys. Lett. B667, 1 (2008). 
18. W. F. L. Hollik, Fortschr. Phys. 38, 165 (1990).

19. F. Jegerlehner, Lectures given at the Theoretical Advanced Study Institute in Elementary Particle Physics, (TASI), Boulder, Colo., Jun 3-29, 1990.

20. A. Sirlin, Phys. Rev. D22, 971 (1980).

21. T. Appelquist and J. Carazzone, Phys. Rév. D11, 2856 (1975).

22. H. Flaecher et al., 0811.0009 .

23. J. Erler and P. Langacker, 0807.3023.

24. M. Lindner, Zeit. Phys. C31, 295 (1986).

25. R. S. Chivukula and N. J. Evans, Phys. Lett. B464, 244 (1999), [hep-ph/9907414].

26. T. Hambye and K. Riesselmann, Phys. Rev. D55, 7255 (1997), [hep-ph/9610272].

27. M. Sher, Phys. Rept. 179, 273 (1989).

28. G. Isidori, G. Ridolfi and A. Strumia, Nucl. Phys. B609, 387 (2001), [hep-ph/0104016].

29. C. F. Kolda and H. Murayama, JHEP 07, 035 (2000), [hep-ph/0003170].

30. M. Drees, hep-ph/9611409.

31. S. Dawson, hep-ph/9712464.

32. B. W. Lee, C. Quigg and H. B. Thacker, Phys. Rev. D16, 1519 (1977).

33. M. J. Duncan, G. L. Kane and W. W. Repko, Nucl. Phys. B272, 517 (1986).

34. CERN-LHCC-99-15.

35. CMS, G. L. Bayatian et al., J. Phys. G34, 995 (2007).

36. N. E. Adam et al., 0803.1154.

37. T. Plehn, D. L. Rainwater and D. Zeppenfeld, Phys. Rev. D61, 093005 (2000), [hep-ph/9911385].

38. N. Kauer, T. Plehn, D. L. Rainwater and D. Zeppenfeld, Phys. Lett. B503, 113 (2001), [hep$\mathrm{ph} / 0012351]$.

39. R. Goncalo, f. t. ATLAS and C. Collaborations, 0811.3778.

40. M. Duhrssen et al., Phys. Rev. D70, 113009 (2004), [hep-ph/0406323].

41. A. Belyaev and L. Reina, JHEP 08, 041 (2002), [hep-ph/0205270]. 